\title{
Exact traveling wave solutions of the KP-BBM equation by using the new approach of generalized $\left(G^{\prime} / G\right)$-expansion method
}

\author{
Md Nur Alam ${ }^{1}$ and M Ali Akbar ${ }^{*}$
}

\begin{abstract}
The new approach of the generalized ( $\left.G^{\prime} / G\right)$-expansion method is an effective and powerful mathematical tool in finding exact traveling wave solutions of nonlinear evolution equations (NLEEs) in science, engineering and mathematical physics. In this article, the new approach of the generalized $\left(G^{\prime} / G\right)$-expansion method is applied to construct traveling wave solutions of the Kadomtsev-Petviashvili-Benjamin-Bona-Mahony (KP-BBM) equation. The solutions are expressed in terms of the hyperbolic functions, the trigonometric functions and the rational functions. By means of this scheme, we found some new traveling wave solutions of the above mentioned equation.
\end{abstract}

Keywords: New generalized (G'/G)-expansion method; KP-BBM equation; Exact solutions; Nonlinear partial differential equation; Homogeneous balance; Traveling wave solutions; Solitary wave solutions

Mathematics subject classification: 35C07; 35C08; 35P99

\section{Introduction}

The world around us is inherently nonlinear and NLEEs are widely used as models to describe the complex physical phenomena. The exact traveling wave solutions of NLEEs play a vital role in nonlinear science and engineering. Therefore, investigating traveling wave solutions is becoming increasingly attractive in nonlinear sciences day by day. However, not all equations posed of these models are solvable. As a result, many new techniques have been successfully developed by diverse group of scientists, such as, the rank analysis method (Feng 2000), the complex hyperbolic function method (Zayed et al. 2006; Chow 1995), the generalized Riccati equation method (Yan and Zhang 2001; Porubov 1996), the Jacobi elliptic function method (Chen and Wang 2005; Xu 2006; Yusufoglu and Bekir 2008; Zayed et al. 2004a), the ansatz method (Hu 2001a, 2001b), the Adomian decomposition method (Wazwaz 2002), the He's homotopy perturbation method (Ganji and Rafei 2006; Ganji 2006; Ganji et al. 2007), the homogeneous balance method (Wang 1995, 1996; Zayed et al. 2004b), the inverse

\footnotetext{
* Correspondence: ali_math74@yahoo.com

${ }^{2}$ Department of Applied Mathematics, University of Rajshahi, Rahjshahi, Bangladesh

Full list of author information is available at the end of the article
}

scattering transform method (Ablowitz and Clarkson 1991), the Darboux transformation method (Matveev and Salle 1991), the Backlund transformation method (Miura 1978), the $\left(G^{\prime} / G\right)$-expansion method (Wang et al. 2008; Akbar et al. 2012a, 2012b, 2012c, 2012d, 2012e; Bekir 2008; Zayed 2009; Zhang et al. 2008), the improved $\left(G^{\prime} / G\right)$-expansion method (Zhang et al. 2010), the modified simple equation method (Jawad et al. 2010; Khan et al. 2013; Zayed and Ibrahim 2012), the Expfunction method (He and Wu 2006; Akbar and Ali 2012; Mohyud-Din et al. 2010), the tanh-function method (Malfliet 1992; Fan 2000), the sine-cosine method (Wazwaz 2004, 2005; Bibi and Mohyud-Din 2013), the first integral method (Feng 2002; Tascan and Bekir 2010) etc.

Recently, the new generalized $\left(G^{\prime} / G\right)$ expansion method has been initiated by Naher and Abdullah (2013). The significance of the new generalized $\left(G^{\prime} / G\right)$ expansion method is that one can treat the nonlinear problems by essentially linear method. Moreover, it transforms a nonlinear evolution equation to a simple algebraic computation. The merits of the new generalized $\left(G^{\prime} / G\right)$ expansion method over the other methods are that it gives more general solutions with some free parameters and it handles NLEEs in a direct manner with no requirement for initial/boundary conditions or initial trial function at the outset. 
Our aim in this paper is to present an application of the new generalized $\left(G^{\prime} / G\right)$ expansion method to solve the KP-BBM equation by using this method for the first time.

The rest of the paper is organized as follows: In Section "Description of the new generalized (G'/G)-expansion method", we give the description of the method. In Section "Application of the method", we exert this method to the KP-BBM equation. In Section "Discussions", Discussions are presented. Conclusions are given in Section "Conclusion".

\section{Description of the new generalized $\left(G^{\prime} / G\right)$-expansion method}

Let us consider a general nonlinear PDE in the form

$$
P\left(u, u_{x}, u_{t}, u_{x x}, u_{x t}, u_{t t}, \cdots\right)
$$

where $u=u(x, t)$ is an unknown function, $P$ is a polynomial in $u(x, t)$ and its derivatives wherein the highest order derivatives and nonlinear terms are involved and the subscripts are used for the partial derivatives.

\section{Step 1}

We combine the real variables $x$ and $t$ by a compound variable $\xi$ :

$$
u(x, t)=u(\xi), \quad \xi=x \pm V t
$$

where $V$ is the speed of the traveling wave. The traveling wave transformation (2) converts Eq. (1) into an ordinary differential equation (ODE) for $u=u(\xi)$ :

$$
Q\left(u, u^{\prime}, u^{\prime \prime}, u^{\prime \prime \prime}, \cdots\right)=0
$$

where $Q$ is a polynomial of $\mathrm{u}$ and its derivatives and the superscripts indicate the ordinary derivatives with respect to $\xi$.

\section{Step 2}

According to possibility Eq. (3) can be integrated term by term one or more times, yields constant(s) of integration. The integral constant may be zero, for simplicity.

\section{Step 3}

Suppose the traveling wave solution of Eq. (3) can be expressed as follows:

$$
u(\xi)=\sum_{i=0}^{N} a_{i}(d+H)^{i}+\sum_{i=1}^{N} b_{i}(d+H)^{-i}
$$

where either $a_{N}$ or $b_{N}$ may be zero, but both $a_{N}$ and $b_{N}$ could be zero at a time, $a_{i}(i=0,1,2, \ldots, N)$ and $b_{i}(i=1$,
$2, \cdots, N)$ and $d$ are arbitrary constants to be determined later and $H(\xi)$ is

$$
H(\xi)=\left(G^{\prime} / G\right)
$$

where $G=G(\xi)$ satisfies the following auxiliary equation:

$$
A G G^{\prime \prime}-B G G^{\prime}-E G^{2}-C\left(G^{\prime}\right)^{2}=0,
$$

where the prime stands for derivative with respect to $\xi$; $A, B, C$ and $E$ are real parameters.

\section{Step 4}

To determine the positive integer $N$, taking the homogeneous balance between the highest order nonlinear terms and the derivatives of the highest order appearing in Eq. (3).

\section{Step 5}

Substitute Eq. (4) and Eq. (6) including Eq. (5) into Eq. (3) with the value of $N$ obtained in Step 4, we obtain polynomials in $(d+H)^{N}(N=0,1,2, \cdots)$ and $(d+H)^{-N}$ $(N=0,1,2, \cdots)$. We collect each coefficient of the resulted polynomials and setting them to zero yields a set of algebraic equations for $a_{i}(i=0,1,2, \ldots, N)$ and $b_{i}(i=$ $1,2, \ldots, N), d$ and $V$.

\section{Step 6}

Suppose that the value of the constants $a_{i}(i=0,1,2, \cdots, N)$, $b_{i}(i=1,2, \cdots, N), d$ and $V$ can be found by solving the algebraic equations obtained in Step 5. Since the general solution of Eq. (6) is well known to us, inserting the values of $a_{i}$ $(i=0,1,2, \cdots, N), b_{i}(i=1,2, \cdots, N), d$ and $V$ into Eq. (4), we obtain more general type and new exact traveling wave solutions of the nonlinear partial differential equation (1).

Using the general solution of Eq. (6), we have the following solutions of Eq. (5):

\section{Family 1}

When $B \neq 0, \psi=A-C$ and $\Omega=B^{2}+4 E(A-C)>0$,

$$
H(\xi)=\left(\frac{G^{\prime}}{G}\right)=\frac{B}{2 \psi}+\frac{\sqrt{\Omega}}{2 \psi} \frac{C_{1} \sinh \left(\frac{\sqrt{\Omega}}{2 A} \xi\right)+C_{2} \cosh \left(\frac{\sqrt{\Omega}}{2 A} \xi\right)}{C_{1} \cosh \left(\frac{\sqrt{\Omega}}{2 A} \xi\right)+C_{2} \sinh \left(\frac{\sqrt{\Omega}}{2 A} \xi\right)}
$$

\section{Family 2}

When $B \neq 0, \psi=A-C$ and $\Omega=B^{2}+4 E(A-C)<0$,

$$
H\left(\xi^{\prime}\right)=\left(\frac{G^{\prime}}{G}\right)=\frac{B}{2 \psi}+\frac{\sqrt{-\Omega}}{2 \psi} \frac{-C_{1} \sin \left(\frac{\sqrt{-\Omega}}{2 A} \xi\right)+C_{2} \cos \left(\frac{\sqrt{-\Omega}}{2 A} \xi\right)}{C_{1} \cos \left(\frac{\sqrt{-\Omega}}{2 A} \xi\right)+C_{2} \sin \left(\frac{\sqrt{-\Omega}}{2 A} \xi\right)}
$$




\section{Family 3}

When $B \neq 0, \psi=A-C$ and $\Omega=B^{2}+4 E(A-C)=0$,

$$
H(\xi)=\left(\frac{G^{\prime}}{G}\right)=\frac{B}{2 \psi}+\frac{C_{2}}{C_{1}+C_{2} \xi}
$$

\section{Family 4}

When $B=0, \psi=A-C$ and $\Delta=\psi E>0$,

$$
H(\xi)=\left(\frac{G^{\prime}}{G}\right)=\frac{\sqrt{\Delta}}{\psi} \frac{C_{1} \sinh \left(\frac{\sqrt{\Delta}}{A} \xi\right)+C_{2} \cosh \left(\frac{\sqrt{\Delta}}{A} \xi\right)}{C_{1} \cosh \left(\frac{\sqrt{\Delta}}{A} \xi\right)+C_{2} \sinh \left(\frac{\sqrt{\Delta}}{A} \xi\right)}
$$

\section{Family 5}

When $B=0, \psi=A-C$ and $\Delta=\psi E<0$,

$$
H(\xi)=\left(\frac{G^{\prime}}{G}\right)=\frac{\sqrt{-\Delta}}{\psi} \frac{-C_{1} \sin \left(\frac{\sqrt{-\Delta}}{A} \xi\right)+C_{2} \cos \left(\frac{\sqrt{-\Delta}}{A} \xi\right)}{C_{1} \cos \left(\frac{\sqrt{-\Delta}}{A} \xi\right)+C_{2} \sin \left(\frac{\sqrt{-\Delta}}{A} \xi\right)}
$$

\section{Application of the method}

In this section, we will bring to bear the new generalized $\left(G^{\prime} / G\right)$ expansion method to construct new and more general traveling wave solutions of the KP-BBM equation. Let us consider the KP-BBM equation

$$
\left(u_{t}+u_{x}-a\left(u^{2}\right)_{x}-b u_{x x t}\right)_{x}+k u_{y y}=0
$$

Now, we use the wave transformation (2) into the Eq. (12), which yields

$$
-V u^{\prime \prime}+u^{\prime \prime}-2 a\left(u^{\prime}\right)^{2}-2 a u u^{\prime \prime}+b V u^{(4)}+k u^{\prime \prime}=0 .
$$

Integrating Eq. (13) twice with respect to $\xi$, we obtain

$$
P+(1-V+k) u+b V u^{/ /}-2 a u^{2}=0
$$

where $P$ is an integral constant which is to be determined.

Taking the homogeneous balance between $u^{2}$ and $u^{\prime \prime}$ in Eq. (14), we obtain $N=2$. Therefore, the solution of Eq. (14) is of the form:

$$
\begin{aligned}
u(\xi)= & a_{0}+a_{1}(d+H)+a_{2}(d+H)^{2} \\
& +b_{1}(d+H)^{-1}+b_{2}(d+H)^{-2}
\end{aligned}
$$

where $a_{0}, a_{1}, a_{2}, b_{1}, b_{2}$ and $d$ are constants to be determined.

Substituting Eq. (15) together with Eqs. (5) and (6) into Eq. (14), the left-hand side is converted into polynomials in $(d+H)^{N}(N=0,1,2, \ldots \ldots)$ and $(d+H)^{-N}(N=1,2, \ldots)$. We collect each coefficient of these resulted polynomials and setting them zero yields a set of simultaneous algebraic equations (for simplicity the equations are not presented here) for $a_{0}, a_{1}, a_{2}, b_{1}, b_{2} d, P$ and $V$. Solving these algebraic equations with the help of symbolic computation software Maple, we obtain following:

\section{Set 1}

$P=0, V=V, d=d, a_{1}=0, a_{2}=0$,

$$
\begin{aligned}
a_{0}= & \frac{1}{2 a A^{2}}\left(12 b V d^{2} \psi^{2}+A^{2} k+A^{2}-V A^{2}+12 b V B d \psi\right. \\
& \left.-8 b V E \psi+b V B^{2}\right), \\
b_{1}= & -\frac{6 b V}{a A^{2}}\left(2 d^{3} \psi^{2}+3 B d^{2} \psi+2 E d \psi+B^{2} d-E B\right) \\
b_{2}= & \frac{6 b V}{a A^{2}}\left(d^{4} \psi^{2}+2 B d^{3} \psi-2 E d^{2} \psi-2 B d E+B^{2} d^{2}+E^{2}\right) .
\end{aligned}
$$

where $\psi=A-C, V, d, A, B, C, E$ are free parameters.

\section{Set 2}

$P=0, V=V, d=d, b_{1}=0, b_{2}=0$,

$$
\begin{aligned}
a_{1}=-\frac{6 b V}{a A^{2}}\left(2 d \psi^{2}+B \psi\right), \\
a_{0}=\frac{1}{2 a A^{2}}\left(12 b V d^{2} \psi^{2}+A^{2} k+A^{2}-V A^{2}+12 b V B d \psi\right. \\
\left.-8 b V E \psi+b V B^{2}\right), a_{2}=\frac{6 b V \psi^{2}}{a A^{2}},
\end{aligned}
$$

where $\psi=A-C, V, d, A, B, C, E$ are free parameters.

\section{Set 3}

$P=0, V=V, d=\frac{-B}{2 \psi}, a_{1}=0, b_{1}=0$,

$$
\begin{aligned}
& a_{0}=-\frac{1}{2 a A^{2}}\left(-A^{2} k-A^{2}+A^{2} V+8 b V E \psi+2 b V B^{2}\right), \\
& a_{2}=\frac{6 b V \psi^{2}}{a A^{2}}, a_{1}=0, V=V, \\
& b_{2}=\frac{3 b V}{8 a A^{2} \psi^{2}}\left(16 E^{2} \psi^{2}+8 E B^{2} \psi+B^{4}\right),
\end{aligned}
$$

where $\psi=A-C, V, A, B, C, E$ are free parameters.

For set 1, substituting Eq. (16) into Eq. (15), along with Eq. (7) and simplifying, yields following traveling 
wave solutions (if $C_{1}=0$ but $C_{2} \neq 0 ; C_{2}=0$ but $C_{1} \neq 0$ ) respectively:

$$
\begin{aligned}
u_{1_{1}}(\xi)= & a_{0}+b_{1}\left(d+\frac{B}{2 \psi}+\frac{\sqrt{\Omega}}{2 \psi} \operatorname{coth}\left(\frac{\sqrt{\Omega}}{2 A} \xi\right)\right)^{-1} \\
& +b_{2}\left(d+\frac{B}{2 \psi}+\frac{\sqrt{\Omega}}{2 \psi} \operatorname{coth}\left(\frac{\sqrt{\Omega}}{2 A} \xi\right)\right)^{-2}, \\
u_{1_{2}}(\xi)= & a_{0}+b_{1}\left(d+\frac{B}{2 \psi}+\frac{\sqrt{\Omega}}{2 \psi} \tanh \left(\frac{\sqrt{\Omega}}{2 A} \xi\right)\right)^{-1} \\
& +b_{2}\left(d+\frac{B}{2 \psi}+\frac{\sqrt{\Omega}}{2 \psi} \tanh \left(\frac{\sqrt{\Omega}}{2 A} \xi\right)\right)^{-2}
\end{aligned}
$$

Substituting Eq. (16) into Eq. (15), along with Eq. (8) and simplifying, the exact solutions become (if $C_{1}=0$ but $C_{2} \neq 0 ; C_{2}=0$ but $C_{1} \neq 0$ ):

$$
\begin{aligned}
u_{1_{3}}(\xi)= & a_{0}+b_{1}\left(d+\frac{B}{2 \psi}+\frac{\sqrt{-\Omega}}{2 \psi} \cot \left(\frac{\sqrt{-\Omega}}{2 A} \xi\right)\right)^{-1} \\
& +b_{2}\left(d+\frac{B}{2 \psi}+\frac{\sqrt{-\Omega}}{2 \psi} \cot \left(\frac{\sqrt{-\Omega}}{2 A} \xi\right)\right)^{-2} \\
u_{1_{4}}(\xi)= & a_{0}+b_{1}\left(d+\frac{B}{2 \psi}-\frac{\sqrt{-\Omega}}{2 \psi} \tan \left(\frac{\sqrt{-\Omega}}{2 A} \xi\right)\right)^{-1} \\
& +b_{2}\left(d+\frac{B}{2 \psi}-\frac{\sqrt{-\Omega}}{2 \psi} \tan \left(\frac{\sqrt{-\Omega}}{2 A} \xi\right)\right)^{-2}
\end{aligned}
$$

Substituting Eq. (16) into Eq. (15), together with Eq. (9) and simplifying, the obtained solution becomes:

$$
\begin{aligned}
u_{1_{5}}(\xi)= & a_{0}+b_{1}\left(d+\frac{B}{2 \psi}+\frac{C_{2}}{C_{1}+C_{2} \xi}\right)^{-1}+b_{2}\left(d+\frac{B}{2 \psi}\right. \\
& \left.+\frac{C_{2}}{C_{1}+C_{2} \xi}\right)^{-2}
\end{aligned}
$$

Substituting Eq. (16) into Eq. (15), along with Eq. (10) and simplifying, we obtain following traveling wave solutions (if $C_{1}=0$ but $C_{2} \neq 0 ; C_{2}=0$ but $C_{1} \neq 0$ ):

$$
\begin{aligned}
u_{1_{6}}(\xi)= & a_{0}+b_{1}\left(d+\frac{\sqrt{\Delta}}{\psi} \operatorname{coth}\left(\frac{\sqrt{\Delta}}{A} \xi\right)\right)^{-1} \\
& +b_{2}\left(d+\frac{\sqrt{\Delta}}{\psi} \operatorname{coth}\left(\frac{\sqrt{\Delta}}{A} \xi\right)\right)^{-2}, \\
u_{1_{7}}(\xi)= & a_{0}+b_{1}\left(d+\frac{\sqrt{\Delta}}{\psi} \tanh \left(\frac{\sqrt{\Delta}}{A} \xi\right)\right)^{-1} \\
& +b_{2}\left(d+\frac{\sqrt{\Delta}}{\psi} \tanh \left(\frac{\sqrt{\Delta}}{A} \xi\right)\right)^{-2}
\end{aligned}
$$

Substituting Eq. (16) into Eq. (15), together with Eq. (11) and simplifying, the obtained exact solutions become (if $C_{1}=0$ but $C_{2} \neq 0 ; C_{2}=0$ but $C_{1} \neq 0$ ) respectively:

$$
\begin{aligned}
u_{1_{8}}(\xi)= & a_{0}+b_{1}\left(d+\frac{\sqrt{-\Delta}}{\psi} \cot \left(\frac{\sqrt{-\Delta}}{A} \xi\right)\right)^{-1} \\
& +b_{2}\left(d+\frac{\sqrt{-\Delta}}{\psi} \cot \left(\frac{\sqrt{-\Delta}}{A} \xi\right)\right)^{-2} \\
u_{1_{9}}(\xi)= & a_{0}+b_{1}\left(d-\frac{\sqrt{-\Delta}}{\psi} \tan \left(\frac{\sqrt{-\Delta}}{A} \xi\right)\right)^{-1} \\
& +b_{2}\left(d-\frac{\sqrt{-\Delta}}{\psi} \tan \left(\frac{\sqrt{-\Delta}}{A} \xi\right)\right)^{-2}
\end{aligned}
$$

where $\xi=x-V t$.

Similarly, for set 2, substituting Eq. (17) into Eq. (15), along with Eq. (7) and simplifying, the traveling wave solutions become (if $C_{1}=0$ but $C_{2} \neq 0 ; C_{2}=0$ but $C_{1} \neq 0$ ) respectively:

$$
\begin{aligned}
u_{2_{1}}(\xi)= & \frac{1}{2 a A^{2}}\left(b V\left(B^{2}-8 E \psi\right)+A^{2}(k-V+1)\right. \\
& \left.+3 b V \Omega \operatorname{coth}^{2}\left(\frac{\sqrt{\Omega}}{2 A} \xi\right)\right) \\
u_{2_{2}}(\xi)= & \frac{1}{2 a A^{2}}\left(b V\left(B^{2}-8 E \psi\right)+A^{2}(k-V+1)\right. \\
& \left.+3 b V \Omega \tanh ^{2}\left(\frac{\sqrt{\Omega}}{2 A} \xi\right)\right),
\end{aligned}
$$

Substituting Eq. (17) into Eq. (15), along with Eq. (8) and simplifying, yields exact solutions (if $C_{1}=0$ but $C_{2} \neq$ $0 ; C_{2}=0$ but $C_{1} \neq 0$ ) respectively:

$$
\begin{aligned}
u_{2_{3}}(\xi)= & \frac{1}{2 a A^{2}}\left(b V\left(B^{2}-8 E \psi\right)+A^{2}(k-V+1)\right. \\
& \left.-3 b V \Omega \cot ^{2}\left(\frac{\sqrt{-\Omega}}{2 A} \xi\right)\right) \\
u_{2_{4}}(\xi)= & \frac{1}{2 a A^{2}}\left(b V\left(B^{2}-8 E \psi\right)+A^{2}(k-V+1)\right. \\
& \left.-3 b V \Omega \tan ^{2}\left(\frac{\sqrt{-\Omega}}{2 A} \xi\right)\right)
\end{aligned}
$$

Substituting Eq. (17) into Eq. (15), along with Eq. (9) and simplifying, our obtained solution becomes:

$$
\begin{aligned}
u_{25}(\xi)= & \frac{1}{2 a A^{2}}\left(A^{2}(k-V+1)-2 b V\left(B^{2}+4 E \psi\right)\right. \\
& \left.+12 b V \psi^{2}\left(\frac{C_{2}}{C_{1}+C_{2} \xi}\right)^{2}\right),
\end{aligned}
$$

Substituting Eq. (17) into Eq. (15), together with Eq. (10) and simplifying, yields following traveling 
wave solutions (if $C_{1}=0$ but $C_{2} \neq 0 ; C_{2}=0$ but $C_{1} \neq 0$ ) respectively:

$$
\begin{aligned}
u_{2_{6}}(\xi)= & \frac{1}{2 a A^{2}}\left(b V\left(B^{2}-8 E \psi\right)+A^{2}(k-V+1)\right. \\
& \left.-12 b V \sqrt{\Delta}\left(B \operatorname{coth}\left(\frac{\sqrt{\Delta}}{A} \xi\right)-\sqrt{\Delta} \operatorname{coth}^{2}\left(\frac{\sqrt{\Delta}}{A} \xi\right)\right)\right), \\
u_{27}(\xi)= & \frac{1}{2 a A^{2}}\left(b V\left(B^{2}-8 E \psi\right)+A^{2}(k-V+1)\right. \\
& \left.-12 b V \sqrt{\Delta}\left(B \tanh \left(\frac{\sqrt{\Delta}}{A} \xi\right)-\sqrt{\Delta} \tanh ^{2}\left(\frac{\sqrt{\Delta}}{A} \xi\right)\right)\right),
\end{aligned}
$$

Substituting Eq. (17) into Eq. (15), along with Eq. (11) and simplifying, our exact solutions become (if $C_{1}=0$ but $C_{2} \neq 0 ; C_{2}=0$ but $C_{1} \neq 0$ ) respectively:

$$
\begin{aligned}
u_{28}(\xi)= & \frac{1}{2 a A^{2}}\left(b V\left(B^{2}-8 E \psi\right)+A^{2}(k-V+1)\right. \\
& \left.-12 b V \sqrt{\Delta}\left(i B \cot \left(\frac{\sqrt{-\Delta}}{A} \xi\right)+\sqrt{\Delta} \cot ^{2}\left(\frac{\sqrt{-\Delta}}{A} \xi\right)\right)\right), \\
u_{29}(\xi)= & \frac{1}{2 a A^{2}}\left(b V\left(B^{2}-8 E \psi\right)+A^{2}(k-V+1)\right. \\
& +12 b V \sqrt{\Delta}\left(i B \tan \left(\frac{\sqrt{-\Delta}}{A} \xi\right)-\sqrt{\Delta} \tan ^{2}\left(\frac{\sqrt{-\Delta}}{A} \xi\right)\right),
\end{aligned}
$$

where $\xi=x-V t$.

Similarly, For set 3, substituting Eq. (18) into Eq. (15), together with Eq. (7) and simplifying, yields following traveling wave solutions (if $C_{1}=0$ but $C_{2} \neq 0 ; C_{2}=0$ but $\left.C_{1} \neq 0\right)$ respectively:

$$
\begin{aligned}
& u_{3_{1}}(\xi)=a_{0}+\frac{3 b V \Omega}{2 a A^{2}} \operatorname{coth}^{2}\left(\frac{\sqrt{\Omega}}{2 A} \xi\right)+\frac{4 b_{2} \psi^{2}}{\Omega} \tanh ^{2}\left(\frac{\sqrt{\Omega}}{2 A} \xi\right), \\
& u_{3_{2}}(\xi)=a_{0}+\frac{3 b V \Omega}{2 a A^{2}} \tanh ^{2}\left(\frac{\sqrt{\Omega}}{2 A} \xi\right)+\frac{4 b_{2} \psi^{2}}{\Omega} \operatorname{coth}^{2}\left(\frac{\sqrt{\Omega}}{2 A} \xi\right),
\end{aligned}
$$

Substituting Eq. (18) into Eq. (15), along with Eq. (8) and simplifying, we obtain following solutions (if $C_{1}=0$ but $C_{2} \neq 0 ; C_{2}=0$ but $C_{1} \neq 0$ ) respectively:

$$
\begin{aligned}
& u_{3_{3}}(\xi)=a_{0}-\frac{3 b V \Omega}{2 a A^{2}} \cot ^{2}\left(\frac{\sqrt{-\Omega}}{2 A} \xi\right)-\frac{4 b_{2} \psi^{2}}{\Omega} \tan ^{2}\left(\frac{\sqrt{-\Omega}}{2 A} \xi\right) \\
& u_{3_{4}}(\xi)=a_{0}-\frac{3 b V \Omega}{2 a A^{2}} \tan ^{2}\left(\frac{\sqrt{-\Omega}}{2 A} \xi\right)-\frac{4 b_{2} \psi^{2}}{\Omega} \cot ^{2}\left(\frac{\sqrt{-\Omega}}{2 A} \xi\right)
\end{aligned}
$$

Substituting Eq. (18) into Eq. (15), along with Eq. (9) and simplifying, our obtained solution becomes:

$$
u_{35}(\xi)=a_{0}+\frac{6 b V \psi^{2}}{a A^{2}}\left(\frac{C_{2}}{C_{1}+C_{2} \xi}\right)^{2}+b_{2}\left(\frac{C_{2}}{C_{1}+C_{2} \xi}\right)^{-2},
$$

Substituting Eq. (18) into Eq. (15), along with Eq. (10) and simplifying, yields following exact traveling wave solutions (if $C_{1}=0$ but $C_{2} \neq 0 ; C_{2}=0$ but $C_{1} \neq 0$ ) respectively:

$$
\begin{aligned}
u_{3_{6}}(\xi)= & a_{0}+\frac{6 b V \psi^{2}}{a A^{2}}\left(\frac{-B}{2 \psi}+\frac{\sqrt{\Delta}}{\psi} \operatorname{coth}\left(\frac{\sqrt{\Delta}}{A} \xi\right)\right)^{2} \\
& +b_{2}\left(\frac{-B}{2 \psi}+\frac{\sqrt{\Delta}}{\psi} \operatorname{coth}\left(\frac{\sqrt{\Delta}}{A} \xi\right)\right)^{-2} \\
u_{37}(\xi)= & a_{0}+\frac{6 b V \psi^{2}}{a A^{2}}\left(\frac{-B}{2 \psi}+\frac{\sqrt{\Delta}}{\psi} \tanh \left(\frac{\sqrt{\Delta}}{A} \xi\right)\right)^{2} \\
& +b_{2}\left(\frac{-B}{2 \psi}+\frac{\sqrt{\Delta}}{\psi} \tanh \left(\frac{\sqrt{\Delta}}{A} \xi\right)\right)^{-2}
\end{aligned}
$$

Substituting Eq. (18) into Eq. (15), along with Eq. (11) and simplifying, the obtained solutions become (if $C_{1}=0$ but $C_{2} \neq 0 ; C_{2}=0$ but $C_{1} \neq 0$ ) respectively:

$$
\begin{aligned}
u_{3_{8}}(\xi)= & a_{0}+\frac{6 b V \psi^{2}}{a A^{2}}\left(\frac{-B}{2 \psi}+\frac{\sqrt{-\Delta}}{\psi} \cot \left(\frac{\sqrt{-\Delta}}{A} \xi\right)\right)^{2} \\
& +b_{2}\left(\frac{-B}{2 \psi}+\frac{\sqrt{-\Delta}}{\psi} \cot \left(\frac{\sqrt{-\Delta}}{A} \xi\right)\right)^{-2} \\
u_{39}(\xi)= & a_{0}+\frac{6 b V \psi^{2}}{a A^{2}}\left(\frac{-B}{2 \psi}-\frac{\sqrt{-\Delta}}{\psi} \tan \left(\frac{\sqrt{-\Delta}}{A} \xi\right)\right)^{2} \\
& +b_{2}\left(\frac{-B}{2 \psi}-\frac{\sqrt{-\Delta}}{\psi} \tan \left(\frac{\sqrt{-\Delta}}{A} \xi\right)\right)^{-2}
\end{aligned}
$$

where $\xi=x-V t$.

\section{Remark}

The solutions obtained in this article have been checked by putting them back into the original equation and found correct.

\section{Discussions}

The advantages and validity of the method over the basic $\left(G^{\prime} / G\right)$-expansion method have been discussed in the following.

\section{Advantages}

The crucial advantage of the new generalized $\left(G^{\prime} / G\right)$ expansion method over the basic $\left(G^{\prime} / G\right)$-expansion method is that the method provides more general and large amount of new exact traveling wave solutions with several free parameters. The exact solutions have its great importance to expose the inner mechanism of the complex physical phenomena. Apart from the physical application, the close-form solutions of nonlinear evolution equations assist the numerical solvers to compare the accuracy of their results and help them in the stability analysis. 


\section{Validity}

Feng and Zheng (2010) investigated the well-established KP-BBM equation to obtain exact solutions via the basic $\left(G^{\prime} / G\right)$-expansion method and achieved only three solutions (A.1),(A.2),(A.3) (see Appendix). On the other hand, twenty seven solutions are constructed of this equation by applying the new approach of generalized $\left(G^{\prime} / G\right)$-expansion method. They used the linear ordinary differential equation as an auxiliary equation and traveling wave solutions presented in the form $u(\xi)=\sum_{i=0}^{m} a_{i}\left(G^{\prime} / G\right)^{i}$, where $a_{m} \neq 0$. It is noteworthy to point out that some of our solutions are coincided with the solutions obtained by Feng and Zheng (2010) if the parameters are taken particular values, which validate our solutions.

\section{Conclusion}

The new generalized $\left(G^{\prime} / G\right)$-expansion method established by Naher and Abdullah has successfully been implemented to construct new and more general exact traveling wave solutions of the KP-BBM equation. The method offers solutions with free parameters that might be important to explain some complex physical phenomena. Comparing the currently proposed method with other methods, such as $\left(G^{\prime} / G\right)$-expansion method, the Exp-function method and the modified simple equation method, we might conclude that the exact solutions to Eq. (12) can be investigated by simple and systematic way. This study shows that the new generalized $\left(G^{\prime} / G\right)$-expansion method is quite efficient and practically well suited to be used in finding exact solutions of NLEEs. Also, we observe that the new generalized $\left(G^{\prime} / G\right)$-expansion method is straightforward and can be applied to many other nonlinear evolution equations.

\section{Appendix}

Feng and Zheng's solutions (Feng and Zheng 2010)

Feng and Zheng (2010) established exact solutions of the well-known the KP-BBM equation by using the basic $\left(G^{\prime} / G\right)$-expansion method which are as follows:

When $\lambda^{2}-4 \mu>0$,

$$
\begin{aligned}
u_{1}= & a_{0}-\frac{a_{2}}{4} \lambda^{2} \\
& +\frac{a_{2}}{4}\left(\lambda^{2}-4 \mu\right)\left(\frac{C_{1} \sinh \frac{1}{2} \sqrt{\lambda^{2}-4 \mu} \xi+C_{2} \cosh \frac{1}{2} \sqrt{\lambda^{2}-4 \mu \xi}}{C_{1} \cosh \frac{1}{2} \sqrt{\lambda^{2}-4 \mu} \xi+C_{2} \sinh \frac{1}{2} \sqrt{\lambda^{2}-4 \mu \xi}}\right)^{2},
\end{aligned}
$$

where $\xi=x+y-\frac{1}{6} \frac{a a_{2}}{b} t$ and $C_{1}, C_{2}$ are arbitrary constants.
When $\lambda^{2}-4 \mu<0$,

$$
\begin{aligned}
u_{2}= & a_{0}-\frac{a_{2}}{4} \lambda^{2} \\
& +\frac{a_{2}}{4}\left(4 \mu-\lambda^{2}\right)\left(\frac{-C_{1} \sin \frac{1}{2} \sqrt{4 \mu-\lambda^{2}} \xi+C_{2} \cos \frac{1}{2} \sqrt{4 \mu-\lambda^{2}} \xi}{C_{1} \cos \frac{1}{2} \sqrt{4 \mu-\lambda^{2}} \xi+C_{2} \sin \frac{1}{2} \sqrt{4 \mu-\lambda^{2}} \xi}\right)^{2},
\end{aligned}
$$

where $\xi=x+y-\frac{1}{6} \frac{a a_{2}}{b} t$ and $C_{1}, C_{2}$ are arbitrary constants.

$$
\begin{aligned}
& \text { When } \lambda^{2}-4 \mu=0 \text {, } \\
& \qquad u_{5}=-\frac{1}{4} \lambda^{2} a_{2}+\frac{a_{2} C_{2}{ }^{2}}{\left(C_{1}+C_{2} \xi\right)^{2}}+a_{0},
\end{aligned}
$$

where $\xi=x+y-\frac{1}{6} \frac{a a_{2}}{b} t$ and $C_{1}, C_{2}$ are arbitrary constants.

\section{Competing interests}

The authors declare that they have no competing interests.

\section{Authors' contributions}

Both the authors, viz MNA and MAA, with the consultation of each other carried out this work and drafted the manuscript together. Both the authors read and approved the final manuscript.

\section{Acknowledgements}

The authors would like to express their sincere thanks to the anonymous referees for their valuable comments and suggestions. The authors also would like to convey their appreciation to Md. Tarik-ul Islam, Professor, Department of English, University of Rajshahi, Bangladesh for his assistance in editing the English language.

\section{Author details}

'Department of Mathematics, Pabna University of Science and Technology, Pabna, Bangladesh. ${ }^{2}$ Department of Applied Mathematics, University of Rajshahi, Rahjshahi, Bangladesh.

Received: 13 September 2013 Accepted: 13 November 2013 Published: 19 November 2013

\section{References}

Ablowitz MJ, Clarkson PA (1991) Soliton, nonlinear evolution equations and inverse scattering. Cambridge University Press, New York

Akbar MA, Ali NHM (2012) New solitary and periodic solutions of nonlinear evolution equation by Exp-function method. World Appl Sci J 17(12):1603-1610

Akbar MA, Ali NHM, Zayed EME (2012a) Abundant exact traveling wave solutions of the generalized Bretherton equation via $\left(G^{\prime} / G\right)$-expansion method. Commun Theor Phys 57:173-178

Akbar MA, Ali NHM, Zayed EME M (2012b) A generalized and improved ( $\left.G^{\prime} / G\right)$ expansion method for nonlinear evolution equations. Math Prob Engr vol. 2012:22 pages. doi: 10.1155/2012/459879

Akbar MA, Ali NHM, Mohyud-Din ST (2012c) The alternative ( $\left.G^{\prime} / G\right)$-expansion method with generalized Riccati equation: application to fifth order $(1+1)$ dimensional Caudrey-Dodd-Gibbon equation. Int J Phys Sci 7(5):743-752

Akbar MA, Ali NHM, Mohyud-Din ST (2012d) Some new exact traveling wave solutions to the $(3+1)$-dimensional Kadomtsev-Petviashvili equation. World Appl Sci J 16(11):1551-1558

Akbar MA, Ali NHM, Mohyud-Din ST (2012e) Further exact traveling wave solutions to the $(2+1)$-dimensional Boussinesq and Kadomtsev-Petviashvili equation. J Comput Anal Appl 15(3):557-571

Bekir A (2008) Application of the ( $\left.G^{\prime} / G\right)$-expansion method for nonlinear evolution equations. Phys Lett A 372:3400-3406

Bibi S, Mohyud-Din ST (2013) Traveling wave solutions of KdVs using sine-cosine method. J Assn Arab Univ Basic Appl Sci. doi: org/10.1016/j.jaubas.2013.03.006 (in press) 
Chen Y, Wang Q (2005) Extended Jacobi elliptic function rational expansion method and abundant families of Jacobi elliptic functions solutions to $(1+1)$-dimensional dispersive long wave equation. Chaos Soliton Fract $24: 745-757$

Chow KW (1995) A class of exact periodic solutions of nonlinear envelope equation. J Math Phys 36:4125-4137

Fan E (2000) Extended tanh-function method and its application to nonlinear equations. Phys Lett A 277(4-5):212-218

Feng $X$ (2000) Exploratory approach to explicit solution of nonlinear evolutions equations. Int J Theo Phys 39:207-222

Feng Z (2002) The first-integral method to study the Burgers-Korteweg-de Vries equation. J Phys A 35(2):343-349

Feng Q, Zheng B (2010) Traveling wave solutions for three nonlinear equations by $\left(G^{\prime} / G\right)$-expansion method. WSEAS Trans Computers 9(3):1109-2750

Ganji DD (2006) The application of He's homotopy perturbation method to nonlinear equations arising in heat transfer. Phys Lett A 355:137-141

Ganji DD, Rafei M (2006) Solitary wave solutions for a generalized Hirota-Satsuma coupled KdV equation by homotopy perturbation method. Phys Lett $A$ 356:131-137

Ganji DD, Afrouzi GA, Talarposhti RA (2007) Application of variational iteration method and homotopy perturbation method for nonlinear heat diffusion and heat transfer equations. Phys Lett A 368:450-457

He JH, Wu XH (2006) Exp-function method for nonlinear wave equations. Chaos Soliton Fract 30:700-708

Hu JL (2001a) A new method for finding exact traveling wave solutions to nonlinear partial differential equations. Phys Lett A 286:175-179

Hu JL (2001b) Explicit solutions to three nonlinear physical models. Phys Lett A 287:81-89

Jawad AJM, Petkovic MD, Biswas A (2010) Modified simple equation method for nonlinear evolution equations. Appl Math Comput 217:869-877

Khan K, Akbar MA, Ali NHM (2013) The modified simple equation method for exact and solitary wave solutions of nonlinear evolution equation: the GZKBBM equation and right handed non-commutative Burger equations. ISRN Math Phys Vol 2013(2013):Article ID 146704. 5 pages

Malfliet W (1992) Solitary wave solutions of nonlinear wave equations. Am J Phys 60(7):650-654

Matveev VB, Salle MA (1991) Darboux transformation and solitons. Springer, Berlin

Miura MR (1978) Backlund transformation. Springer, Berlin

Mohyud-din ST, Noor MA, Noor KI (2010) Exp-function method for traveling wave solutions of modified Zakharov-Kuznetsov equation. J King Saud Univ 22:213-216

Naher H, Abdullah FA (2013) New approach of $\left(G^{\prime} / G\right)$-expansion method and new approach of generalized $\left(G^{\prime} / G\right)$-expansion method for nonlinear evolution equation. AIP Adv 3:032116. doi: 10. 1063/1.4794947

Porubov AV (1996) Periodical solution to the nonlinear dissipative equation for surface waves in a convecting liquid layer. Phys Lett A 221:391-394

Tascan F, Bekir A (2010) Applications of the first integral method to nonlinear evolution equations. Chin Phys B 19(8):080201

Wang M (1995) Solitary wave solutions for variant Boussinesq equations. Phys Lett A 199:169-172

Wang ML (1996) Exact solutions for a compound KdV-Burgers equation. Phys Lett A 213:279-287

Wang M, Li X, Zhang J (2008) The ( $\left.G^{\prime} / G\right)$-expansion method and traveling wave solutions of nonlinear evolution equations in mathematical physics. Phys Lett A 372:417-423

Wazwaz AM (2002) Partial Differential equations: Method and Applications. Taylor and Francis, London

Wazwaz AM (2004) A sine-cosine method for handing nonlinear wave equations. Math Comput Model 40:499-508

Wazwaz AM (2005) The tanh and the sine-cosine methods for the complex modified KdV and the generalized KdV equations. Comput Math Appl 49(7-8):1101-1112

Xu G (2006) An elliptic equation method and its applications in nonlinear evolution equations. Chaos Soliton Fract 29:942-947

Yan Z, Zhang H (2001) New explicit solitary wave solutions and periodic wave solutions for Whitham Broer-Kaup equation in shallow water. Phys Lett A 285(5-6):355-362

Yusufoglu E, Bekir A (2008) Exact solution of coupled nonlinear evolution equations. Chaos Soliton Fract 37:842-848
Zayed EME (2009) The ( $\left.G^{\prime} / G\right)$-expansion method and its applications to some nonlinear evolution equations in the mathematical physics. J Appl Math Comput 30:89-103

Zayed EME, Ibrahim SAH (2012) Exact solutions of nonlinear evolution equations in mathematical physics using the modified simple equation method. Chin Phys Lett 29(06):060201

Zayed EME, Zedan HA, Gepreel KA (2004a) On the solitary wave solutions for nonlinear Euler equations. Appl Anal 83:1101-1132

Zayed EME, Zedan HA, Gepreel KA (2004b) On the solitary wave solutions for nonlinear Hirota-Sasuma coupled KDV equations. Chaos Soliton Fract 22:285-303

Zayed EME, Abourabia AM, Gepreel KA, Horbaty MM (2006) On the rational solitary wave solutions for the nonlinear Hirota Satsuma coupled KdV system. Appl Anal 85:751-768

Zhang S, Tong J, Wang W (2008) A generalized $\left(G^{\prime} / G\right)$-expansion method for the mKdV equation with variable coefficients. Phys Lett A 372:2254-2257

Zhang J, Jiang F, Zhao X (2010) An improved ( $\left.G^{\prime} / G\right)$-expansion method for solving nonlinear evolution equations. Int J Com Math 87(8):1716-1725

\section{doi:10.1186/2193-1801-2-617}

Cite this article as: Alam and Akbar: Exact traveling wave solutions of the KP-BBM equation by using the new approach of generalized $\left(G^{\prime} / G\right)$ expansion method. SpringerPlus 2013 2:617.

\section{Submit your manuscript to a SpringerOpen ${ }^{\odot}$ journal and benefit from:}

- Convenient online submission

- Rigorous peer review

- Immediate publication on acceptance

- Open access: articles freely available online

- High visibility within the field

- Retaining the copyright to your article

Submit your next manuscript at $>$ springeropen.com 